\title{
Is Roux-en-Y gastric bypass advantageous? - surgical outcomes in obese patients with type-2 diabetes after gastric bypass versus sleeve gastrectomy, a matched retrospective study
}

\author{
Hong-Wei Zhang ${ }^{1}$, Xiao-Dong Han ${ }^{2}$, Wei-Jie Liu ${ }^{2}$, Hao-Yong Yu ${ }^{3}$, Pin Zhang ${ }^{2}$, Zhong-Qi Mao ${ }^{1}$ \\ ${ }^{1}$ Department of General Surgery, The First Affiliated Hospital of Soochow University, Suzhou 215006, China; ${ }^{2}$ Department of Metabolic \& Bariatric \\ Surgery, Shanghai Jiao Tong University Affiliated Sixth People's Hospital, Shanghai 200233, China; ${ }^{3}$ Department of Endocrinology and Metabolism, \\ Shanghai Jiao Tong University Affiliated Sixth People's Hospital, Shanghai Diabetes Institute, Shanghai Clinical Center of Diabetes, Shanghai Key \\ Laboratory of Diabetes Mellitus, Shanghai 200233, China \\ Contributions: (I) Conception and design: HW Zhang, ZQ Mao; (II) Administrative support: P Zhang; (III) Provision of study materials or patients: \\ XD Han, P Zhang; (IV) Collection and assembly of data: HW Zhang, XD Han, WJ Liu; (V) Data analysis and interpretation: HW Zhang, HY Yu; (VI) \\ Manuscript writing: All authors; (VII) Final approval of manuscript: All authors. \\ Correspondence to: Zhong-Qi Mao. Department of General Surgery, The First Affiliated Hospital of Soochow University, No. 899, Rd. Pinhai, Suzhou \\ 215006, China. Email: zqmaosuzhou@hotmail.com.
}

Background: There are few comparative studies of the clinical outcomes after Roux-en-Y gastric bypass (RYGB) and sleeve gastrectomy (SG). The aim of the study was to compare diabetes- and metabolic disorder-related outcomes following RYGB and SG, based on data for matched participants.

Methods: This was a retrospective matched study using data from 2011-2018. Patients with type-2 diabetes undergoing RYGB ( $n=35$ ) were matched with up to 2 RYGB participants $(n=56)$ regarding age, sex, body mass index, hemoglobin A1c level, medication use, diabetes duration, and blood pressure.

Results: All surgeries were performed laparoscopically without complications or malnutrition during 24 months of follow-up. Both surgical procedures achieved excellent diabetes remission and weight loss. RYGB was associated with a significantly higher diabetes medication discontinuation rate 24 months postoperatively (RYGB: $87.5 \%$ vs. SG: $68.6 \%$; $\mathrm{P}<0.05$ ), better reduction in serum cholesterol and low-density lipoprotein-c levels, as well as better diabetes control compared with SG. The incidence of microvascular and macrovascular complications showed no significant difference between two groups.

Conclusions: In this matched retrospective study, although RYGB and SG were both excellent surgeries for treating obesity in patients with type-2 diabetes, RYGB was associated with better results compared with SG regarding dyslipidemia remission and metabolic disorder-related medication reduction.

Keywords: Type 2 diabetes mellitus (T2D); Roux-en-Y gastric bypass (RYGB); sleeve gastrectomy (SG); lipid profiles; diabetes vascular complications

Submitted Oct 31, 2019. Accepted for publication Dec 24, 2019.

doi: $10.21037 /$ atm.2020.02.08

View this article at: http://dx.doi.org/10.21037/atm.2020.02.08

\section{Introduction}

All current bariatric procedures are considered effective in the treatment of morbid obesity and its related comorbidities, compared with nonsurgical interventions $(1,2)$. The profound effect of type 2 diabetes mellitus (T2D) remission suggests that bariatric and metabolic surgery is a successful treatment for T2D. Recent data showed that the number of sleeve gastrectomies (SG) has increased rapidly and SG is now the most-frequently performed surgical procedure worldwide, followed by Roux-en-Y gastric bypass (RYGB). In Asia, $>60 \%$ of metabolic surgical procedures are SG (3). Although RYGB is still considered the most popular 


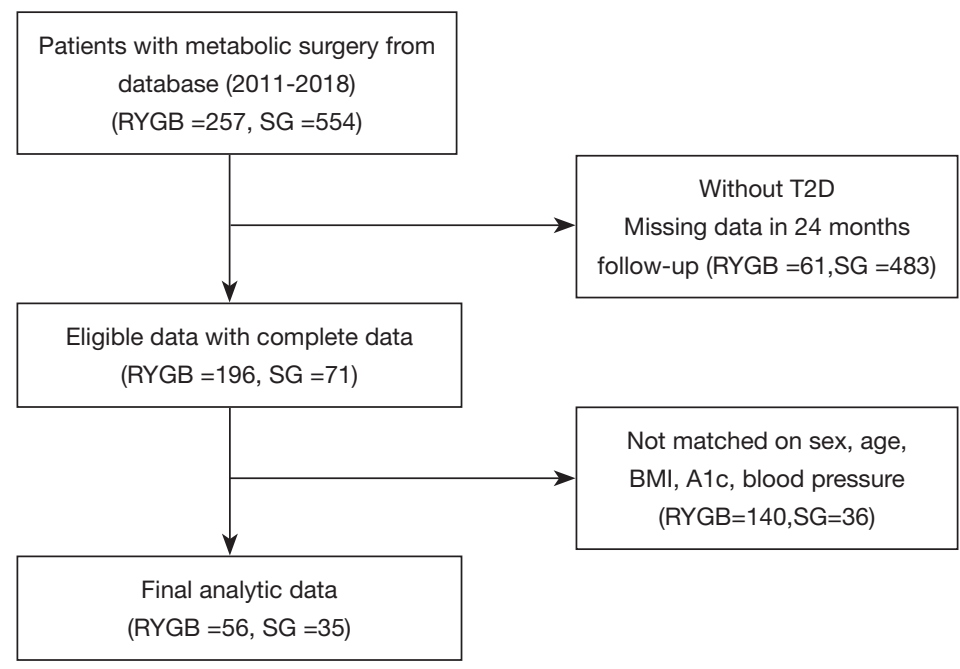

Figure 1 Flow diagram for identification of eligible patients with T2D and the sample size of RYGB and SG patients. T2D, type 2 diabetes mellitus; RYGB, Roux-en-Y gastric bypass; SG, sleeve gastrectomy.

surgical procedure to treat $\mathrm{T} 2 \mathrm{D}$ with obesity, one study showed that SG achieved similar results regarding T2D remission compared with RYGB (4). The major anatomical difference between the two procedures is exclusion of the proximal small intestine with RYGB while SG involves complete resection of the stomach fundus. The mechanism of T2D remission following RYGB involves several factors such as changes in hormone secretion and nutrient absorption, possible changes in serum bile acids, and composition of the microbiome. The beneficial effects of SG are considered to involve many of these factors as well; however, because SG is a less complex procedure, it is considered as safe but with less complications compared with RYGB. This raises the question whether SG will replace RYGB and become the most suitable surgical procedure in treating T2D.

Considering how few studies have compared the T2D remission effect between RYGB and SG in matched patients, the objective of the present study was to investigate the changes in diabetes- and metabolic disorder-related issues in patients with obesity who underwent SG compared with well-matched individuals who underwent RYGB.

\section{Methods}

\section{Setting}

This was a retrospective study of adults who underwent bariatric and metabolic surgery between January 2011 and December 2018 in two hospitals. The human research review board of each institution approved the study, and all patients provided written informed consent.

\section{Study participants}

Following the latest guidelines in our country for metabolic surgery, the inclusion criteria were as follows: (I) patients with T2D with adequate islet function; (II) age 16-65 years; (III) body mass index (BMI) $25-27.5 \mathrm{~kg} / \mathrm{m}^{2}$ with poorlycontrolled T2D and more than two symptoms of metabolic syndrome or T2D complications; and (IV) BMI $>27.5 \mathrm{~kg} / \mathrm{m}^{2}$ with poorly-controlled T2D. Patients with established diagnoses of type 1 diabetes mellitus, latent autoimmune diabetes in adulthood, malignancy, debilitating disease, unresolved psychiatric illness, or substance abuse were excluded from the study.

After selecting patients for possible inclusion, we applied the following exclusion criteria according to information collected during $<24$ months of continuous enrollment after surgery. For each patient undergoing SG, we identified up to 2 matched RYGB participants according to the study site, sex, age ( \pm 5 years), BMI $\left( \pm 2.5 \mathrm{~kg} / \mathrm{m}^{2}\right)$, hemoglobin A1c level $( \pm 1.0 \%)$, blood pressure $( \pm 10 \mathrm{mmHg})$, and metabolic disorder-related medications. Patients undergoing RYGB could be used as a control for only 1 patient undergoing SG (matching without replacement). The flow diagram for identifying eligible patients is shown in Figure 1. We enrolled 56 patients undergoing RYGB and 35 patients undergoing SG. 
Table 1 Baseline characteristics in RYGB and SG groups

\begin{tabular}{|c|c|c|c|}
\hline Parameters & RYGB $(n=56)$ & $S G(n=35)$ & $P$ value \\
\hline T2D duration (yr) & $5.86 \pm 4.70$ & $6.29 \pm 4.26$ & 0.180 \\
\hline Gender (M/F) & $26(\mathrm{M}) ; 30(\mathrm{~F})$ & $17(\mathrm{M}) ; 18(\mathrm{~F})$ & 0.842 \\
\hline \multicolumn{4}{|l|}{ BMI } \\
\hline 30 to 35 & $10(\mathrm{M}) ; 13(\mathrm{~F})$ & 8 (M); 3 (F) & 0.218 \\
\hline$\geq 35$ & $15(\mathrm{M}) ; 16(\mathrm{~F})$ & $8(\mathrm{M}) ; 14(\mathrm{~F})$ & 0.556 \\
\hline Hypertension (n/total) & $26 / 56$ & $16 / 35$ & 0.947 \\
\hline Dyslipidemia (n/total) & $43 / 56$ & $24 / 35$ & 0.386 \\
\hline
\end{tabular}

RYGB, Roux-en-Y gastric bypass; SG, sleeve gastrectomy; T2D, type 2 diabetes mellitus; BMI, body mass index; M, male; F, female.

\section{Surgical procedure}

The surgical procedures for RYGB and SG were as described previously. Briefly, RYGB involves creating a gastric pouch of approximately $30 \mathrm{~mL}$ volume, with a length of $100 \mathrm{~cm}$ for both the alimentary limb and biliopancreatic $\operatorname{limb}(5)$. With a 37-French bougie inside the gastric lumen, SG was performed with the short gastric vessels 5 -cm proximal to the pylorus and up to $1 \mathrm{~cm}$ from the angle of His (6).

\section{Anthropometric and biochemical evaluations}

Anthropometric evaluations, namely, BMI and blood pressure, were recorded before and after surgery. Patients' medical history, and current medications for diabetes, hypertension, and dyslipidemia were also recorded.

Biochemical parameters were evaluated by obtaining serum samples after an overnight fast and were as follows: fasting glucose, postprandial glucose followed by an oral glucose tolerance test, fasting C-peptide level, fasting insulin level, A1c, serum total cholesterol (Tc), triglyceride, high-density lipoprotein cholesterol (HDL-c), and lowdensity lipoprotein cholesterol (LDL-c). Nutrition status was evaluated with serum hemoglobin, calcium level, 25-dihydroxy vitamin D3 and ferritin levels. Arteriosclerosis risk index was calculated as the (Tc - HDL-c)/HDL-c ratio. Insulin resistance levels were measured by the homeostasis model assessment of insulin resistance (HOMA-IR) using the formula, $\mathrm{mU} / \mathrm{mmol} / \mathrm{L}^{2}=$ fasting insulin $(\mathrm{mU} / \mathrm{L}) \times$ fasting glucose $(\mathrm{mmol} / \mathrm{L}) / 22.5$.
The incidence calculations use the composite end points microvascular complications (retinopathy, nephropathy or neuropathy, which ever came first) and macrovascular complications (legs, heart, and brain, whichever came first).

\section{Statistical analysis}

All statistics were calculated using SPSS statistical software (version 22.0; IBM Corp., Armonk, NY, USA). Contingency tables of the categorical variables were analyzed by the Kruskal-Wallis test. Data are presented as the mean \pm standard deviation or median (interquartile range). Statistical significance was defined as $\mathrm{P}<0.05$.

\section{Results}

\section{Participants}

There were 91 patients involved in this study, with 56 in the RYGB group and 35 in the SG group. All surgeries were performed laparoscopically without mortality or major complications. No severe malnutrition or vitamin or mineral deficiencies were observed during follow-up. Patients' baseline characteristics are shown in Table 1; there were no significant differences between the groups.

\section{RYGB vs. SG with anthropometric and biochemical results}

Tables 2 and 3 provide the anthropometric and metabolic disorder-related biochemical results between the RYGB and SG groups. Mean BMI, diastolic blood pressure, A1c, 
Table 2 Characteristics and diabetes related index changes before and after surgery

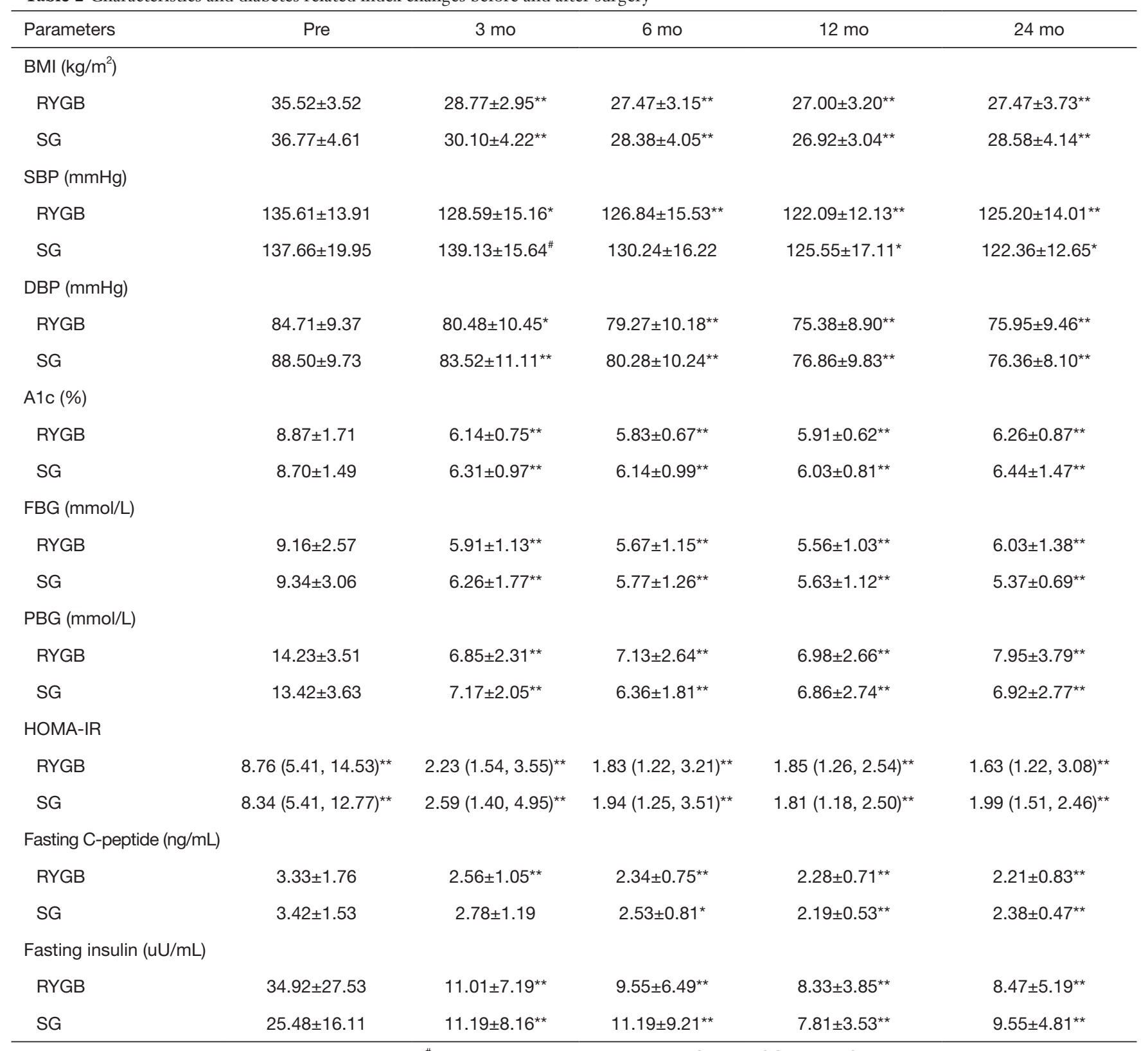

*, $\mathrm{P}<0.05$, ${ }^{*}, \mathrm{P}<0.01$ : compared to pre-operation; *, $\mathrm{P}<0.05$ : compared between RYGB and SG group. SBP, systolic blood pressure; DBP, diastolic blood pressure; A1C, hemoglobin A1c; FBG, fasting plasma glucose; PBG, postprandial glucose; HOMA-IR, Homeostasis Model Assessment-Insulin Resistance; RYGB, Roux-en-Y gastric bypass; SG, sleeve gastrectomy.

fasting and postprandial glucose, fasting C-peptide and insulin levels, serum triglycerides, and HDL-c and HOMAIR decreased significantly decreased after surgery, with no significant differences between the groups.

Systolic blood pressure in the RYGB group decreased significantly 3 months after RYGB and 12 months after SG. Systolic blood pressures in the SG group were higher than in the RYGB group 3 months postoperatively.

Serum Tc and LDL-c levels in the RYGB group decreased significantly postoperatively; however, there was no significant difference in the SG group pre- and postoperatively. Mean Tc and LDL-c levels in the RYGB group were lower than in the SG group. Because the arteriosclerosis risk index was calculated using Tc and HDL-c levels, the arteriosclerosis 
Table 3 Lipid profiles and arteriosclerosis risk index changes before and after surgery

\begin{tabular}{|c|c|c|c|c|c|}
\hline Parameters & Pre & $3 \mathrm{mo}$ & $6 \mathrm{mo}$ & $12 \mathrm{mo}$ & $24 \mathrm{mo}$ \\
\hline RYGB & $5.30 \pm 1.18$ & $4.55 \pm 0.92^{\star \star}$ & $4.30 \pm 0.90^{\star \star}$ & $4.22 \pm 0.96^{\star \star}$ & $4.39 \pm 1.04^{\star *}$ \\
\hline SG & $4.86 \pm 1.11$ & $5.41 \pm 0.90^{\# \#}$ & $5.07 \pm 1.06^{\# \#}$ & $5.02 \pm 1.30^{\# \#}$ & $5.21 \pm 1.34^{\#}$ \\
\hline \multicolumn{6}{|l|}{$\mathrm{Tg}(\mathrm{mmol} / \mathrm{L})$} \\
\hline SG & $2.62 \pm 1.53$ & $1.61 \pm 0.73^{\star \star}$ & $1.31 \pm 0.53^{\star \star}$ & $1.22 \pm 0.42^{\star \star}$ & $1.35 \pm 0.55^{\star *}$ \\
\hline \multicolumn{6}{|c|}{ HDL-c (mmol/L) } \\
\hline RYGB & $0.98 \pm 0.19$ & $1.01 \pm 0.21$ & $1.14 \pm 0.21^{\star \star}$ & $1.23 \pm 0.24^{\star \star}$ & $1.20 \pm 0.28^{\star \star}$ \\
\hline SG & $1.01 \pm 0.18$ & $1.10 \pm 0.25$ & $1.18 \pm 0.24^{\star \star}$ & $1.31 \pm 0.25^{\star \star}$ & $1.21 \pm 0.28^{\star *}$ \\
\hline$S G$ & $2.86 \pm 0.86^{\#}$ & $3.43 \pm 0.68^{\star \# \#}$ & $3.17 \pm 0.92^{\# \#}$ & $2.95 \pm 1.04^{\#}$ & $3.10 \pm 0.86^{\#}$ \\
\hline \multicolumn{6}{|l|}{ ASI } \\
\hline RYGB & $4.61 \pm 1.90$ & $3.64 \pm 1.13^{\star \star}$ & $2.86 \pm 0.97^{\star \star}$ & $2.53 \pm 0.89^{\star *}$ & $2.81 \pm 1.11^{\star \star}$ \\
\hline$S G$ & $3.86 \pm 1.07^{\#}$ & $4.11 \pm 1.41^{\#}$ & $3.48 \pm 1.39^{\#}$ & $2.91 \pm 1.09^{\star \star}$ & $3.40 \pm 0.98^{\#}$ \\
\hline
\end{tabular}

*, $\mathrm{P}<0.05$, ${ }^{* \star}, \mathrm{P}<0.01$ : compared to pre-operation;, $\mathrm{P}<0.05$, ${ }^{\# \#, ~} \mathrm{P}<0.01$ : compared between RYGB and SG group. Tc, total cholesterol; $\mathrm{Tg}$, total triglycerides; HDL-c, high-density lipoprotein cholesterol; LDL-c, low-density lipoprotein cholesterol; ASI, arteriosclerosis index; RYGB, Roux-en-Y gastric bypass; SG, sleeve gastrectomy.

Table 4 Alc remission rate in RYGB and SG groups

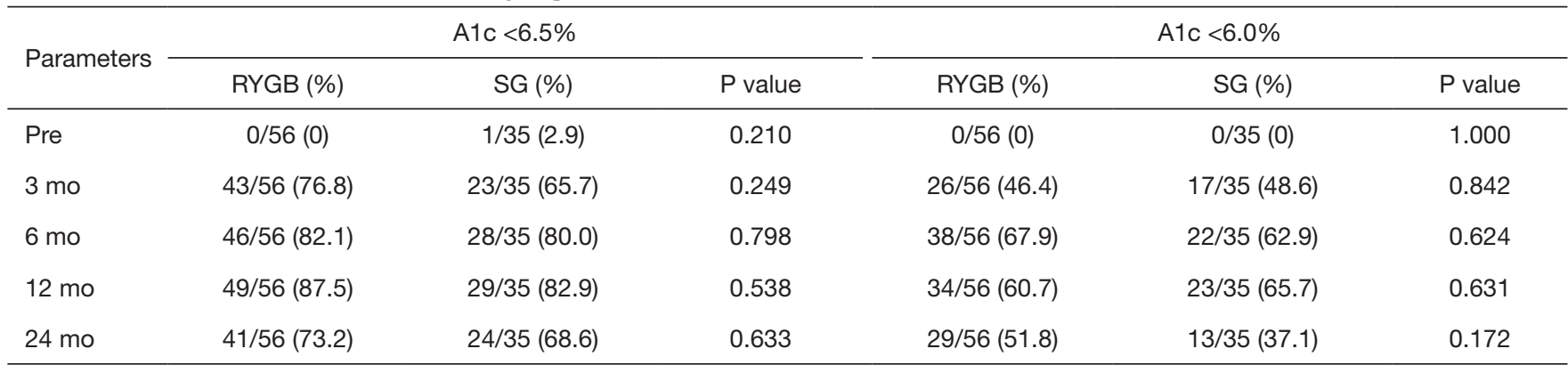

RYGB, Roux-en-Y gastric bypass; SG, sleeve gastrectomy.

index in the RYGB group decreased significantly, and the index value was also lower than in the SG group.

The $\mathrm{T} 2 \mathrm{D}$ remission rates according to A1c levels are shown in Table 4. According to the latest guidelines for the prevention and control of type 2 diabetes in our country, which recommend achieving an A1c $<6.5 \%$, remission rates did not differ significantly between the two groups. However, under the more strict standards for diabetes control of an A1c $<6 \%$, patients in the RYGB group achieved a $51.8 \%$ remission rate compared with $37.1 \%$ in the SG group.

\section{RYGB vs. SG regarding medications for diabetes and metabolic disorders}

Postoperatively, the use of medications for metabolic disorders (hypertension and dyslipidemia) and diabetes, including insulin, decreased significantly from baseline in both surgical groups. 
A

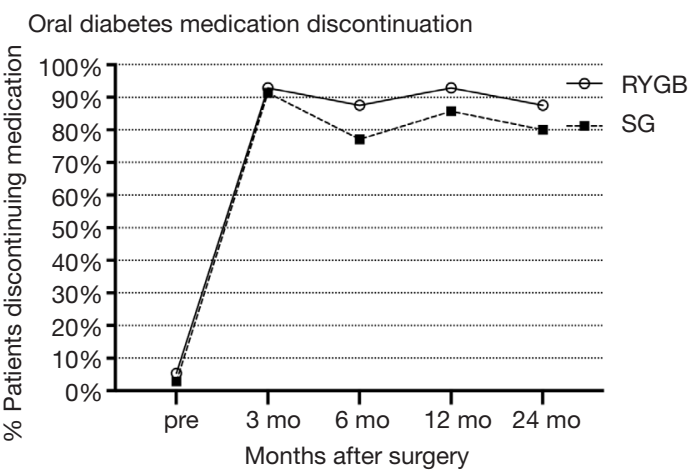

C

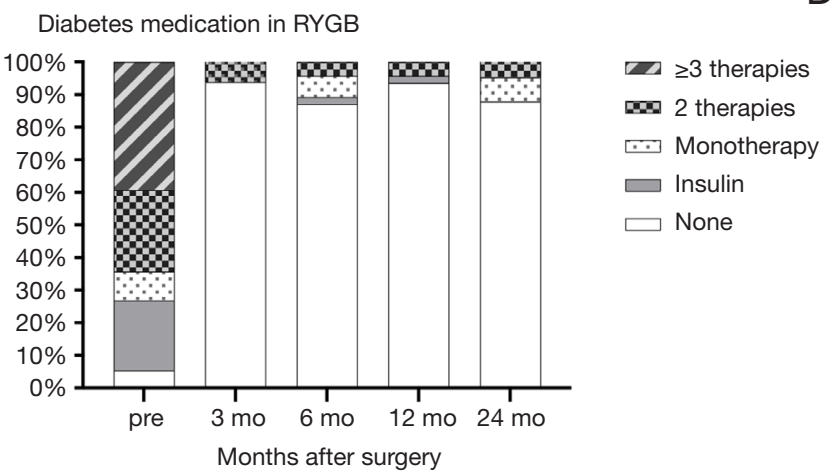

B

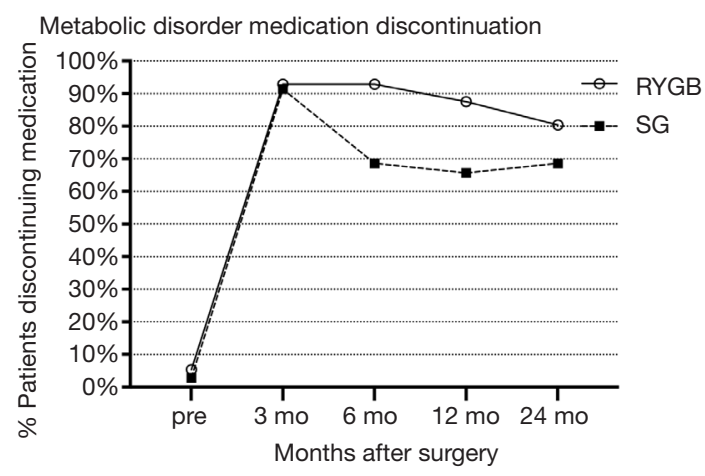

Diabetes medication in SG

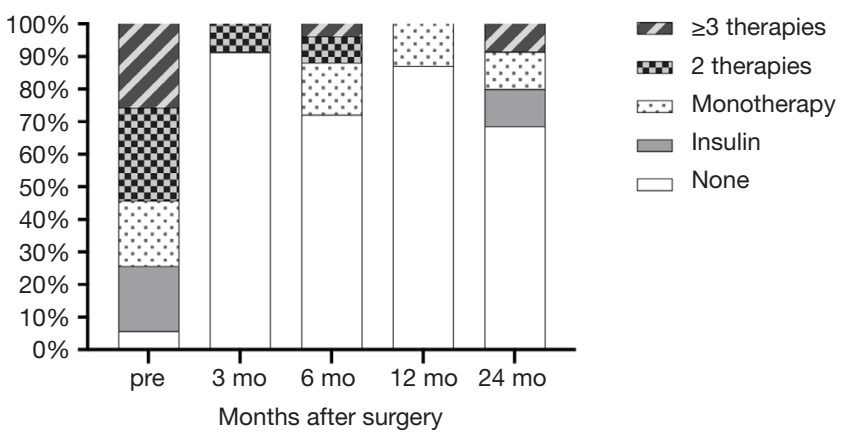

Figure 2 Mean changes in diabetes and metabolic disorder-related medication among matched RYGB and SG patients. Shown are the mean oral T2D medication discontinuation rate between RYGB and SG (A), metabolic disorder (hypertension and dyslipidemia) medication discontinuation rates (B). (C) and (D) were oral T2D medication and insulin use status in RYGB and SG patients. Less T2D medication use in the RYGB group than in the SG group 24 months postoperatively $(\mathrm{P}<0.05)$.

The overall oral diabetes medication discontinuation rates in the RYGB group from the preoperative rate to 24 months postoperatively were $5.4 \%, 92.9 \%, 87.5 \%$, $92.9 \%$, and $87.5 \%$, respectively, and in the SG group, overall diabetes medication discontinuation rates were $2.9 \%$, $91.4 \%, 77.1 \%, 85.7 \%$, and $80 \%$, respectively. There was no significant difference between the two groups for each follow-up time-point. Comparing the same time-points, the metabolic disorder (hypertension and dyslipidemia) medication discontinuation rates were $5.4 \%, 92.9 \%, 92.9 \%$, $87.5 \%$, and $80.4 \%$ respectively, in the RYGB group and $2.9 \%, 91.4 \%, 68.6 \%, 65.7 \%$, and $68.6 \%$, respectively, in the SG group. Metabolic disorder medication discontinuation rates were significantly higher following RYGB $v$ s. following SG 12 months postoperatively ( $\mathrm{P}=0.013)$.

The percentage of patients who were taking no glucoselowering medications, including insulin, was significantly higher in the RYGB group than in the SG group 24 months postoperatively (RYGB: $87.5 \%$ vs. SG: $68.6 \%$; $\mathrm{P}<0.05$ ). Additionally, 3 patients in the $S G$ group required more than 3 types of glucose-lowering medications $v s$. no patients in the RYGB group (Figure 2).

\section{Nutrition status}

Table 5 provides the Nutrition status results between the RYGB and SG groups. Mean serum calcium level, 25-dihydroxy vitamin D3 and ferritin levels showed no difference pre- and postoperatively in each group, there with no significant differences between the groups as well. However, hemoglobin level decreased in the RYGB group 12 and 24 months, mean hemoglobin level were significantly lower following RYGB vs. following SG 24 months postoperatively $(\mathrm{P}=0.038)$. Three patients in RYGB group were diagnosed anemia 12 months postoperatively. Four patients in RYGB group and one in SG 
Table 5 Nutrition status before and after surgery

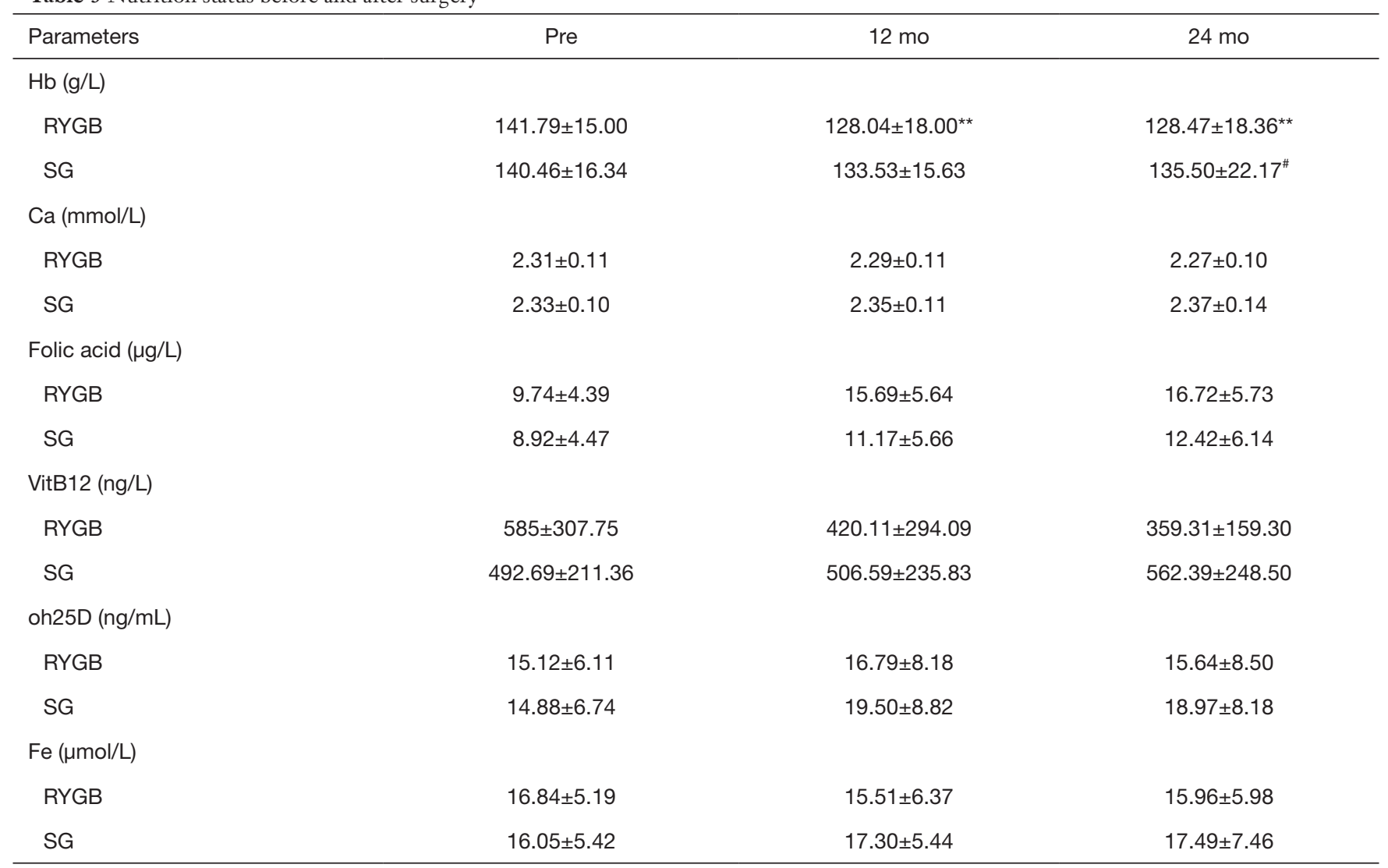

**, P<0.01: compared to pre-operation; ", P<0.05, compared between RYGB and SG group. Hb, hemoglobin; Ca: serum calcium level; oh25D, 25-dihydroxy vitamin D3; Fe, serum ferritin level.

group were diagnosed anemia 24 months postoperatively.

\section{Diabetes complications}

The incidence calculations use the composite end points microvascular complications and macrovascular complications were in Table 6. There was no significant difference between two groups. The composite macrovascular complications rate was $8.9 \%$ in RYGB group while 20\% in SG group 24 months postoperatively.

\section{Discussion}

In this retrospective matched study, we observed significant and sustained reductions in patients' BMI values and rates of diabetes after SG and RYGB. Patients' mean BMI decreased to $<30 \mathrm{~kg} / \mathrm{m}^{2}$ and was maintained to 6 months postoperatively with no significant difference between the two types of metabolic surgery. Both surgeries were associated with remarkable and sustained improvement in diabetes markers while avoiding malnutrition. The degree of weight loss in patients in both the RYGB and SG groups was similar to that reported in a previous study (7). RYGB was associated with an early reduction in blood pressure compared with SG: systolic blood pressure in the RYGB group had decreased by 3 months postoperatively and was significantly decreased 12 months after SG. However, diastolic blood pressure decreased early in both the RYGB and SG groups. One 10-year cohort study suggested comparable effectiveness between SG and RYGB regarding weight loss; however, RYGB was superior to SG in achieving 10-year hypertension remission (8).

After 24 months of follow-up, was saw significant improvement in diabetes status, including a significant reduction in C-peptide levels, serum insulin level, serum glucose, A1c, and HOMA-IR, indicating improved insulin resistance in both groups, and no difference between RYGB and SG. Mullally et al. also showed that short-term 
Table 6 Results of incident microvascular and macrovascular outcomes in RYGB vs. SG groups

\begin{tabular}{|c|c|c|c|}
\hline Parameters & RYGB $(n=56)(\%)$ & $S G(n=35)(\%)$ & $P$ value \\
\hline Composite incident microvascular disease $\mathrm{a}^{\mathrm{a}}$ & 3.6 & 2.9 & 0.676 \\
\hline Incident diabetic retinopathy & 1.8 & 2.9 & 0.692 \\
\hline Incident diabetic nephropathy & 0 & 0 & - \\
\hline Composite incident macrovascular disease ${ }^{b}$ & 3.6 & 8.6 & 0.144 \\
\hline \multicolumn{4}{|l|}{24 months } \\
\hline Composite incident microvascular disease & 12.5 & 11.4 & 0.859 \\
\hline Incident diabetic retinopathy & 5.4 & 2.9 & 0.968 \\
\hline Composite incident macrovascular disease & 8.9 & 20. & 0.230 \\
\hline
\end{tabular}

${ }^{\mathrm{a}}$, indicates the first occurrence of retinopathy, nephropathy or neuropathy; ${ }^{\mathrm{b}}$, indicates the first occurrence of macrovascular complications (legs, heart, and brain)

improvements in beta-cell function using an intravenous glucose-tolerance test were similar between patients undergoing SG vs. RYGB (9). Other studies also confirmed that there was no significant difference between the two procedures, but some randomized clinical trials have shown less weight loss and smaller improvements in A1c or higher use of diabetes medications to achieve similar glycemic control following SG, compared with RYGB $(10,11)$.

Supplemental recommendations for both groups included daily consumption of 2,000 IU of vitamin D3, 1,500$2,000 \mathrm{mg}$ of calcium citrate, $60-80 \mathrm{~g}$ of protein, $350 \mathrm{ug}$ of vitamin B12, and a multivitamin twice a day, which was according to Chinese Society for Metabolic Bariatric Surgery latest guideline. However, more patients in RYGB group had lower serum hemoglobin level and developed anemia compared to SG group. The most efficient regions of iron absorption are the duodenum and proximal jejunum, obviously, these regions are bypassed following RYGB. The decrease in total iron body stores may result in a decrease in erythropoiesis, which may induce a concurrent irondeficient anemia. These biochemical results are not unique as the literature documents, lifelong biochemical follow-up is necessary, especially in RYGB group.

The $\mathrm{T} 2 \mathrm{D}$ remission rate in our study decreased according to A1c levels during the 24-month follow-up. The mean Alc level reached its lowest point 6 months after RYGB and 12 months after SG. Observational studies have shown a similar decreasing remission rate in several middleto long-term follow-ups. Using a cutoff of an A1c of $<6.5 \%$, the remission rate following RYGB in our study was $73.2 \%$ vs. $68.6 \%$ following SG. However, the gap between the two procedures widened when we used the cutoff of an A1c $<6 \%$ : $51.8 \%$ patients achieved remission following RYGB, and only $37.1 \%$ achieved remission following SG. These results showed that RYGB resulted in more strict control of $\mathrm{A} 1 \mathrm{c}$ and diabetes compared with SG.

Patients in both groups showed a significant reduction in the use of diabetes-related medications, including insulin. Compared with patients undergoing SG, the percentage of patients taking no glucose-lowering medications was similar 3 months after RYGB. However, 6 months after surgery, the overall use of diabetes-related medications in the RYGB group was lower than in the SG group, ranging from $5.4-87.8 \%$ for RYGB and $5.7-68.6 \%$ for SG. Patients undergoing RYGB had a larger decrease in their cumulative dosing of diabetes medications and insulin. In a large claims-based, nationwide cohort of bariatric patients with diabetes, those undergoing RYGB were more likely to be able to stop all medications compared with those undergoing SG (12), which also indicated that RYGB was more effective for medication discontinuation $v s$. SG.

A few observational studies have found an association between bariatric surgery and fewer micro and macrovascular events when compared with usual care 
$(13,14)$. With over 15 year-follow-up, the cumulative incidence of microvascular complications was 41.8 per 1,000 person-years (95\% CI, 35.3-49.5) for control patients and 20.6 per 1,000 person-years (95\% CI, 17.0-24.9) in the surgery group [hazard ratio (HR), 0.44 ; $95 \%$ CI, 0.34-0.56; $\mathrm{P}<0.001]$. Macrovascular complications were observed in 44.2 per 1,000 person-years (95\% CI, 37.5-52.1) in control patients and 31.7 per 1,000 person-years (95\% CI, 27.0$37.2)$ for the surgical group (HR, 0.68; 95\% CI, 0.54-0.85; $\mathrm{P}=0.001)$ (15).

In this study, results of incident microvascular and macrovascular outcomes between RYGB and SG groups showed no significant difference. Composite incident macrovascular disease in RYGB group was $8.9 \%$ while in SG group increased to $20 \%$. The limited sample size might be one reason.

The effect of RYGB on Tc and LDL cholesterol was the main differential effect on the lipid profile compared with SG. Although mean Tc and LDL-c levels in the RYGB group were higher than in the SG group preoperatively, patients undergoing RYGB showed a significant reduction compared with those undergoing SG. However, we found no significant differences in triglyceride and HDL-c levels between the two groups. A possible explanation for the superiority of RYGB over SG regarding LDL-c and Tc reduction may be its malabsorptive effect. RYGB was designed with a malabsorptive area nearing a length of $100 \mathrm{~cm}$ for the biliopancreatic limb, while SG was designed as a purely restrictive procedure. Several studies showed similar results, with patients undergoing RYGB achieving better dyslipidemia remission $v s$. SG (16-18).

Metabolic changes following RYGB might include changes in bile acid synthesis because bile acids are synthesized from cholesterol in the liver with the ratelimiting enzyme 7a-hydroxylase conjugated to taurine or glycine, secreted into the bile, discharged into the duodenum, and then efficiently reabsorbed in the terminal ileum and the colon (19). Serum bile acids levels are significantly increased in obese patients 2-4 years after RYGB (20), and RYGB can result in increased circulating levels of bile acids, which is supported by experiments performed in rodents (21). The noticeable weight loss and greater dyslipidemia improvement achieved following RYGB vs. SG decreases the risk of atherosclerosis, myocardial infarction, stroke, cardiovascular events, and cardiovascular mortality.

A recent study showed that SG is the most frequentlyperformed bariatric procedure worldwide and has overtaken RYGB (3). The simpler surgical technique with the promising long-term weight loss following SG compared with RYGB could be the reason. In our country, SG is performed more often than RYGB; however, few studies have compared the results of T2D remission with matched patients. Although SG and RYGB resulted in similar remission of obesity and T2D, in our study, RYGB maintained advantages in lipid profile control and metabolic disorder-related medication reduction. RYGB is still recommended as an excellent procedure for treating obesity and T2D, especially in patients with severe metabolic disorders and those taking multiple types and large dosages of medications preoperatively.

Important limitations of our study include the retrospective design and an inadequate sample size and duration to detect differences in long-term efficacy. Therefore, our results should be regarded as preliminary.

\section{Conclusions}

Despite the limitations in our study, we conclude that RYGB and SG are both excellent surgeries to treat obesity with T2D. RYGB was associated with better results compared with SG regarding dyslipidemia remission and metabolic disorder-related medication reduction. Future randomized prospective studies in this field with long-term follow-up are mandatory to confirm our results.

\section{Acknowledgments}

Funding: This study was supported by Science Foundation of Shanghai Municipal Commission of Health and Family Planning, "A prospective study for evaluating clinical results of treating type 2 diabetes with Roux-en-Y gastric bypass", Grant Number: 201640082; Science Foundation of Shanghai Municipal Commission of Health and Family Planning for Young Scholar, "The mechanism of improving diabetic atherosclerosis by down-regulation of LOX-1 after Roux-en-Y gastric bypass" Grant Number: 20164 Y0145.

\section{Footnote}

Conflicts of Interest: The authors have no conflicts of interest to declare.

Ethical Statement: The authors are accountable for all aspects of the work in ensuring that questions related 
to the accuracy or integrity of any part of the work are appropriately investigated and resolved. The human research review board of each institution approved the study, and all patients provided written informed consent.

Open Access Statement: This is an Open Access article distributed in accordance with the Creative Commons Attribution-NonCommercial-NoDerivs 4.0 International License (CC BY-NC-ND 4.0), which permits the noncommercial replication and distribution of the article with the strict proviso that no changes or edits are made and the original work is properly cited (including links to both the formal publication through the relevant DOI and the license). See: https://creativecommons.org/licenses/by-nc-nd/4.0/.

\section{References}

1. Brito JP, Montori VM, Davis AM. Metabolic Surgery in the Treatment Algorithm for Type 2 Diabetes: A Joint Statement by International Diabetes Organizations. JAMA 2017;317:635-36.

2. Adams TD, Davidson LE, Litwin SE, et al. Weight and Metabolic Outcomes 12 Years after Gastric Bypass. N Engl J Med 2017;377:1143-55.

3. Angrisani L, Santonicola A, Iovino P, et al. Bariatric Surgery and Endoluminal Procedures: IFSO Worldwide Survey 2014. Obes Surg 2017;27:2279-89.

4. Shoar S, Saber AA. Long-term and midterm outcomes of laparoscopic sleeve gastrectomy versus Roux-en-Y gastric bypass: a systematic review and meta-analysis of comparative studies. Surg Obes Relat Dis 2017;13:170-80.

5. Zhang H, Di J, Yu H, et al. The Short-Term Remission of Diabetic Nephropathy After Roux-en-Y Gastric Bypass in Chinese Patients of T2DM with Obesity. Obes Surg 2015;25:1263-70.

6. Di J, Wang C, Zhang P, et al. The middle-term result of laparoscopic sleeve gastrectomy in Chinese obesity patients in a single hospital, with the review of literatures and strategy for gastric stenosis. Ann Transl Med 2018;6:479.

7. Zhang H, Han X, Yu H, et al. Effect of Roux-en-Y Gastric Bypass on Remission of T2D: Medium-Term Follow-up in Chinese Patients with Different BMI Obesity Class. Obes Surg 2017;27:134-42.

8. Jiménez A, Ibarzabal A2, Moizé V, et al. Ten-year outcomes after Roux-en-Y gastric bypass and sleeve gastrectomy: an observational nonrandomized cohort study. Surg Obes Relat Dis 2019;15:382-8.
9. Mullally JA, Febres GJ, Bessler M, et al. Sleeve Gastrectomy and Roux-en-Y Gastric Bypass Achieve Similar Early Improvements in Beta-cell Function in Obese Patients with Type 2 Diabetes. Sci Rep 2019;9:1880.

10. Schauer PR, Bhatt DL, Kirwan J, et al. Bariatric surgery versus intensive medical therapy for diabetes--3-year outcomes. N Engl J Med 2014;370:2002-13.

11. Schauer PR, Bhatt DL, Kirwan JP, et al. Bariatric Surgery versus Intensive Medical Therapy for Diabetes - 5-Year Outcomes. N Engl J Med 2017;376:641-51.

12. Lewis KH, Arterburn DE, Zhang F, et al. Comparative Effectiveness of Vertical Sleeve Gastrectomy Versus Roux-en-y Gastric Bypass for Diabetes Treatment: A Claims-based Cohort Study. Ann Surg 2019. [Epub ahead of print].

13. Fisher DP, Johnson E, Haneuse S, et al. Association Between Bariatric Surgery and Macrovascular Disease Outcomes in Patients With Type 2 Diabetes and Severe Obesity. JAMA 2018;320:1570-82.

14. O'Brien R, Johnson E, Haneuse S, et al. Microvascular Outcomes in Patients With Diabetes After Bariatric Surgery Versus Usual Care. Ann Intern Med 2018,169:300-10.

15. Sjöström L, Peltonen M, Jacobson PA, et al. Association of Bariatric Surgery With Long-term Remission of Type 2 Diabetes and With Microvascular and Macrovascular Complications. JAMA 2014,311:2297-304.

16. Climent E, Benaiges D, Flores-Le Roux JA, et al. Changes in the lipid profile 5 years after bariatric surgery: laparoscopic Roux-en-Y gastric bypass versus laparoscopic sleeve gastrectomy. Surg Obes Relat Dis 2018,14:1099-105.

17. Liaskos C, Koliaki C, Alexiadou K, et al. Rouxen-Y Gastric Bypass Is More Effective than Sleeve Gastrectomy in Improving Postprandial Glycaemia and Lipaemia in Non-diabetic Morbidly Obese Patients: a Short-term Follow-up Analysis. Obes Surg 2018;28:3997-4005.

18. Gómez-Martin JM, Balsa JA, Aracil E, et al. Beneficial changes on plasma apolipoproteins $\mathrm{A}$ and $\mathrm{B}$, high density lipoproteins and oxidized low density lipoproteins in obese women after bariatric surgery: comparison between gastric bypass and sleeve gastrectomy. Lipids Health Dis 2018,17:145.

19. Liu H, Hu C, Zhang X, et al. Role of gut microbiota, bile acids and their cross-talk in the effects of bariatric surgery on obesity and type 2 diabetes. J Diabetes 
Investig 2018;9:13-20.

20. Patti ME, Houten SM, Bianco AC, et al. Serum bile acids are higher in humans with prior gastric bypass: potential contribution to improved glucose and lipid metabolism. Obesity (Silver Spring) 2009;17:1671-7.

Cite this article as: Zhang HW, Han XD, Liu WJ, Yu HY, Zhang P, Mao ZQ. Is Roux-en-Y gastric bypass advantageous? - surgical outcomes in obese patients with type2 diabetes after gastric bypass versus sleeve gastrectomy, a matched retrospective study. Ann Transl Med 2020;8(6):372. doi: 10.21037/atm.2020.02.08
21. Myronovych A, Kirby M, Ryan KK, et al. Vertical sleeve gastrectomy reduces hepatic steatosis while increasing serum bile acids in a weight-loss-independent manner. Obesity 2014,22:390-400. 